\title{
Parámetros de calidad en la etapa de desarrollo y maduración en frutos de dos variedades y un cultivar de mandarina (Citrus reticulata Blanco)
}

\author{
Parameters regarding quality during development and maturation \\ stages in fruit from two varieties of a mandarin orange cultivar (Citrus \\ reticulata Blanco)
}

\author{
Os parâmetros de qualidade na fase de desenvolvimento ematuração dos \\ frutos em duas variedades e cultivares de tangerina (Citrus reticulatabranco)
}

Leonardo Villalba-Campos ${ }^{1}$, Aníbal O. Herrera-Arévalo², Javier Orlando Orduz-Rodríguez ${ }^{3}$

Ingeniero Agrónomo, Facultad de Agronomía. Universidad Nacional de Colombia. Sede Bogotá.

Ingeniero de Alimentos, PhD, Profesor asociado, Facultad de Agronomía. Universidad Nacional de Colombia. Sede Bogotá.

Ingeniero Agrónomo, PhD, Centro de Investigaciones la Libertad. Corpoica.

Email: Ivillalbac@unal.edu.co_

Recibido: Octubre 11 de 2012. Aceptado: Septiembre 13 de 2013.

\section{Resumen}

Se determinó el comportamiento de las variables de calidad del fruto de tres variedades de mandarina $(C$. reticulata Blanco): Dancy, Arrayana y un cultivar experimental denominado LL053; injertadas sobre el patrón Cleopatra (C. reticulata Blanco). Las variables evaluadas fueron peso, firmeza, diámetro (polar y ecuatorial), volumen, sólidos solubles totales (SST), acidez total titulable (ATT), relación de madurez (RM) e índice de color (IC). Con cuatro tratamientos correspondientes a los materiales, se utilizó un diseño completamente al azar (DCA), con cuatro árboles por unidad experimental y cuatro repeticiones. Se realizaron cuatro muestreos de forma aleatoria en la fase II de crecimiento del fruto cada cerca de tres semanas a partir de los 148 días después de antesis y el último en el día 208 dda. En la madurez de consumo se les hizo un muestreo a los 222 dda a la mandarina Dancy y 260 dda para Arrayana y LL053. El índice de relación de madurez (RM) muestra un comportamiento temprano para los frutos de mandarina Dancy, alcanzando la relación 10 (SST/ATT), aproximadamente a los 206 dda; mientras que Arrayana y LL053 lo alcanzaron a los 244 y 252 dda respectivamente. Arrayana presentó los mayores valores de peso de frutos, diámetro ecuatorial, $\mathrm{pH}$ y volumen. El cultivar "LL053" y la variedad "Dancy" exhibieron los mayores porcentajes de contenido de jugo (10\% más que Arrayana) y mayores valores de firmeza. El mayor valor de SST se presentó en el cultivar LL053, mientras que la variedad Dancy presentó los valores menores. El índice de color fue mayor en Arrayana y LL053 a los 260 dda, mientras que la variedad Dancy presentó los frutos más verdes a los 220 dda, alcanzando un color naranja intenso con una mayor velocidad en el tiempo, con el aumento de la RM. El comportamiento temprano de la madurez de consumo de la mandarina Dancy es importante para el desarrollo de la producción de mandarinas en el trópico bajo de Colombia, ya que permite ampliar la época de cosecha y competir con la calidad interna y externa con la fruta importada.

Palabras clave: Características fisicoquímicas, crecimiento y desarrollo, madurez de consumo. 


\begin{abstract}
The pattern of variables was determined regarding the fruit quality of three clementine varieties (Dancy, Arrayana and an experimental cultivar - LL053) grafted onto Cleopatra clementine (C. reticulata Blanco) stock. The variables evaluated in this study were weight, firmness, diameter (polar and equatorial), volume, total soluble solids (TSS), total titratable acidity (TTA), maturity index (MI) and colour index (CI). Treatment involved a completely randomised design (CRD), with four trees per experimental unit and four repetitions concerning the aforementioned materials. Four random samplings were made during fruit growth phase II every three weeks from 148 days post anthesis (DPA) onwards and the last on day 208 DPA. Ripening was sampled on day 222 DPA for the Dancy mandarin and 260 DPA for Arrayana and LL053. MI revealed an early pattern for Dancy mandarin fruit, achieving 10 TSS/TTA ratio around day 206 DPA whilst Arrayana achieved this on day 244 DPA and LL053 on day 252 DPA. Arrayana had greater fruit weight, equatorial diameter, $\mathrm{pH}$ and volume values. The LL053 cultivar and Dancy variety had the highest juice content percentages $(10 \%$ higher than that for Arrayana) and greater firmness values. The LL053 cultivar had a highest TSS value, while the Dancy variety had the lowest value. The CI was higher for Arrayana and LL053 260 DPA, while the Dancy variety had greener fruit 220 DPA, achieving an intense orange colour in a faster way, with increased MI. The Dancy mandarin's early ripening pattern is important for mandarin production in Colombia's tropical lowlands since it allows harvesting time to be prolonged and the fruit to become competitive regarding imported fruit's internal and external quality.
\end{abstract}

Key words: Physicochemical characteristics, growth and development, ripening.

\title{
Resumo
}

Se determino o comportamento das variáveis de qualidade de frutos de três variedades de tangerina (C. reticulata Blanco): Dancy, Arrayan a e experimental cultivar chamado LL053, enxertadosempadrão Cleopatra (C. reticulata Blanco). As variáveis avaliadas foram peso, firmeza, diâmetro (polar e equatorial), volume, Sólidos Solúveis Totais (SST), Acidez Total Titulável (ATT), Relacao de Maturidade (RM) e índice da cor (IC). Com quatro tratamentos correspondentes a os materiais, um delineamento inteiramente casualizado (DIC) com quatro árvores por parcela e quatro repetições. Quatro coletas for am feitas de forma aleatória na fase II de crescimento do fruto cada três semanas a partir dos 148 dias de pois deanteses ea última no dia 208 dda. No amadurecimento de consumo realizou-se uma amostragemà 222 daàtangerina Dancy ea os 260 dda para Arrayana e LL053. O índice de relacao de maturidade (RM) mostra um comportamento cedo para os frutos de tangerina Dancy, a relaçãoatingi-o 10 (SST/ATT), aproximadamente à206 dda; enquanto que Arrayana y LL053 chegouà244 e 252dda, respectivamente. Arrayana apresentou os maiores valores de peso do fruto, diâmetro equatorial, pH e volume. O cultivar "LL053" e variedade "Dancy" exibiram os mai orespercentuais de teor de suco (10\% a mais do que Arrayana) e maiores valores de firmeza. O maior valor de SST é apresentado no cultivar LL053, em quanto a variedade Dancy apresentou os menores valores. O índice dacorfoimaiorna arrayana y LL053 à260dda, em quanto avariedade Dancy presento u os frutos mais verdes à 220dda, atingindo umacorlaranja intenso comuma maiorvelocidade no tempo com o aumento da RM. O comportamento no início do am adurecimento de consumo da tangerina Dancy é importante para o desenvolvimento da produção de tangerina nos trópicos baixos da Colômbia; ja que permite ampliar o período de colheita e competir com a qualidade interna e externa com o fruto importado.

Palavras-chave: propriedades físico-químicas, crescimento e desenvolvimento, amadurecimento do consumo.

\section{Introducción}

El grupo de mandarino común perteneciente a la especie Citrus reticulata Blanco, es el más cultivado a escala mundial por su amplia adaptación a las condiciones climáticas (Anderson, 1996); sin embargo, sus variedades son muy específicas en requerimientos climáticos para producir frutos de buena calidad. Las principales regiones productoras del mundo están ubicadas en el subtrópico entre los $25^{\circ}$ y $40^{\circ}$ de latitud en ambos hemisferios. Cuando estas variedades se cultivan en condiciones tropicales presentan un com- 
portamiento en producción y calidad diferentes al que tienen cuando se cultivan en condiciones del subtrópico (Orduz-Rodríguez, 2007). Las áreas citrícolas en el subtrópico, se caracterizan por presentar temperaturas medias anuales entre los 15 y $20^{\circ} \mathrm{C}$, con inviernos desde leves a severos y heladas ocasionales, mientras que en el trópico se registran temperaturas medias anuales entre los 20 y $30^{\circ} \mathrm{C}$ y las variaciones en producción y calidad están asociadas a los cambios en altitud, desde el nivel del mar hasta la cercanía a las cordilleras (Landanilla, 2008). En condiciones tropicales cerca al Ecuador, donde se cultivan comercialmente los cítricos por debajo de los 2.100 msnm, y no se presentan periodos de reposo por bajas temperaturas, éstas son constantes (a la misma altitud), al igual que el fotoperiodo y la radiación. En estas condiciones, el principal factor climático que influye sobre el comportamiento del crecimiento y desarrollo de las plantas cítricas está determinado por la presencia o ausencia de la precipitación (Orduz-Rodríguez, 2007).

Las condiciones climáticas tienen un efecto significativo sobre la calidad de la fruta, la tasa de crecimiento y el desarrollo (Landanilla, 2008). En el trópico, con el inicio de la temporada lluviosa o la aplicación de riego, se presenta la hidratación de las plantas, se inicia el proceso de desarrollo floral que continúa de forma ininterrumpida hasta el final de la antesis (caída de pétalos); luego se presenta el cuajado, el crecimiento y desarrollo del fruto y finalmente su madurez (Orduz et al., 2009). En los cítricos, el desarrollo del fruto sigue una curva sigmoidal, desde antesis hasta maduración (Davies y Albrigo, 1999; Orduz- Rodríguez et al., 2009), con tres fases diferenciadas: en la primera fase, se evidencia un crecimiento rápido, donde se diferencian todos los tejidos. Esta etapa ocurre en un periodo que abarca el fin de la floración y la caída fisiológica del fruto, por acción de la rápida división celular donde se generan todas las células que forman la fruta madura. En la segunda fase, las células forman diferentes tipos de tejidos (sacos de jugo, albedo, flavedo, entre otros), hay elongación celular y las vesículas se llenan de azúcares, ácidos y agua. En la tercera fase se presenta un lento crecimiento y se producen los cambios relacionados con la maduración del fruto (Agustí et al., 2003) hasta alcanzar un estado de madurez apropiado para consumo (Díaz, 2000). Durante el desarrollo y maduración los cítricos adquieren los atributos de calidad relacionada con propiedades físicas como tamaño, forma, color, textura, número de semillas, entre otras, además de componentes químicos, entre ellos azúcares, ácidos, compuestos volátiles y otras sustancias nutracéuticas como la vitamina C (Iglesias et al., 2007).

Los cítricos son frutos no climatéricos, por tanto, su intensidad respiratoria y producción de etileno no ex- hiben un notable aumento durante su maduración y no presentan cambios marcados en su calidad interna después de la cosecha (Landanilla, 2008).

Las tasas de crecimiento y desarrollo, las características externas y la calidad del fruto están determinadas por las condiciones climáticas de la región y por la variedad cultivada (Orduz et al., 2009). No obstante, la citricultura colombiana posee un escaso número de variedades cultivadas comercialmente (Orduz-Rodríguez, 2003; Orduz-Rodríguez et al., 2011). Dentro de las variedades más cultivadas están la mandarina Oneco en el occidente del país y la mandarina Arrayana en el oriente y centro colombianos, en especial Cundinamarca, Boyacá y Meta (Morales y López, 2007). En menor escala se cultiva la Clementina y la mandarina Dancy (Orduz-Rodríguez y Mateus, 2012); esta última se ha reportado en Santander (Morales y López, 2007).

La citricultura de los Ilanos orientales de Colombia depende fundamentalmente de la variedad Arrayana, lo cual ocasiona una excesiva concentración de cosecha (Orduz-Rodríguez et al., 2011), y representa dificultades y pérdidas importantes para los agricultores (Orduz-Rodríguez y Mateus, 2012). Por tal motivo, se considera que diversificar las variedades ayudaría a estabilizar los precios y a obtener ingresos más constantes (Arana, 2013); de igual forma, permitiría ampliar la época de cosecha, abrir nuevos mercados y evitaría el exceso de fruta en momentos determinados, con la disminución de los precios como se presenta en forma tradicional (Orduz-Rodríguez, 2008; Orduz-Rodríguez y Avella, 2008; Arana, 2013). Para las condiciones actuales de mercado y de producción las propiedades fisicoquímicas de los frutos de cítricos tienen un gran interés, ya que están relacionadas con la percepción de los consumidores y pueden estimular o limitar el desarrollo de la industria de los cítricos (Iglesias et al., 2007).

La mandarina Arrayana es una variedad de origen colombiano, adaptada al trópico bajo (Orduz-Rodríguez et al., 2006). Presenta una sola cosecha importante al final del año en condiciones del piedemonte Ilanero. A causa de esto el total de la producción se acumula en dos meses provocando disminución en el precio, esta situación se repite en otras especies de cítricos (Orduz-Rodríguez et al., 2011).

La mandarina Dancy es la variedad más importante en la Florida (Davies y Albrigo, 1999; Landanilla, 2008). La principal característica de esta variedad, es su elevada tasa de embrionia nucelar (casi del $100 \%$, por tal motivo, cuando Dancy es cultivado a partir de semilla es idéntico a los progenitores y además, presenta un 
periodo juvenil mucho menor en comparación con las naranjas dulces (Davies y Albrigo, 1999).

Otra mandarina, de la cual no se encuentran reportes de cultivos en Colombia es el cultivar LL053 que podría ser una mutación natural aparentemente de Dancy o una introducción a la región de la Orinoquía.

Debido a la escasa información científica sobre el desarrollo y la calidad de los frutos de mandarina que presentan madurez temprana en el trópico, el objetivo de este estudio fue evaluar la calidad de dos variedades (Dancy y Arrayana) y un cultivar de mandarina denominado LL053, injertados sobre el patrón Cleopatra, durante la etapa de desarrollo y maduración de la fruta y su relación con la época de cosecha y comercialización de la producción.

\section{Materiales y métodos}

El estudio se desarrolló entre septiembre de 2011 y enero del 2012. La fase de campo tuvo lugar en el centro de investigación CORPOICA La Libertad, $\left(4^{\circ} 03^{\prime} \mathrm{N}\right.$, $73^{\circ} 29^{\prime} \mathrm{W}$ ), en el departamento del Meta, a una altitud de $336 \mathrm{msnm}$, precipitación anual promedio de 2.921 $\mathrm{mm}$, temperatura media anual de $26^{\circ} \mathrm{C}$ y humedad relativa del $80 \%$. La fase de laboratorio se realizó en los laboratorios de Fisiología Vegetal, Poscosecha y Microbiología del Suelo de la Facultad de Ciencias Agrarias y en los laboratorios del Instituto de Ciencia y Tecnología de Alimentos (ICTA) de la Universidad Nacional de Colombia, sede Bogotá.

Se utilizó un diseño completamente al azar (DCA) con tres tratamientos que correspondieron a los tres materiales vegetales (variedad Dancy y Arrayana" y el cultivar LL053), todos injertados sobre el patrón Cleopatra. Se utilizaron cuatro árboles por unidad experimental, con cuatro repeticiones, para un total de 12 unidades experimentales.

Se realizaron muestreos a los 148, 168, 188, 208 días después de antesis (dda) y un último muestreo en la madurez de consumo 222 dda para "Dancy" y 260 dda para "Arrayana" y "LL053" (Figura 4). Para lo anterior, 12 frutos fueron colectados al azar por material en cada muestreo. Los frutos fueron cosechados en el tercio medio de los árboles. En la interpretación de los resultados y análisis de la información se debe tener en cuenta que Dancy se midió hasta los 222 dda y Arrayana y LL053 hasta los 260 dda debido a las características internas del fruto.

En cada muestreo se determinó peso de los frutos, mediante balanza de precisión con aproximación 0,001 g; firmeza sobre la epidermis usando un penetrómetro digital PCE-PTR200 con aproximación 0,05 N; diámetro polar y ecuatorial, obtenidos entre los polos del fruto y en la zona ecuatorial, respectivamente, medido con un calibrador Vernier; el índice de color (IC) de epidermis se midió con un colorímetro digital marca Minolta, se determinaron los parámetros del sistema CIELab L*, a* y $b^{*}$, con tres lecturas en la parte ecuatorial de cada fruto, y se calculó mediante la fórmula IC $=1000 \times \mathrm{a}^{*} / \mathrm{L}^{*} \mathrm{x}$ $b^{*}$; los sólidos solubles totales (SST) se midieron con un refractómetro digital marca HANNA de rango 0 a $85 \%$ a $20^{\circ} \mathrm{C}$; la acidez total titulable (ATT) (con base en el ácido cítrico) se determinó teniendo en cuenta el volumen de $\mathrm{NaOH}(0,1 \mathrm{~N})$ incorporado en $5 \mathrm{~g}$ de jugo, en una titulación potenciométrica hasta $\mathrm{pH}$ de 8,$2 ;$ y la relación de madurez (RM) se calculó con la relación SST/ATT.

Los datos obtenidos cumplieron con los supuestos de normalidad (Shapiro-Wilk) y homogeneidad de varianzas, por lo tanto se realizó un análisis de varianza (anova) y una prueba de comparación múltiple de Tukey, con un nivel de confianza del 95\% (P $\leq 0,05)$. Todo mediante la utilización del software SAS v. 9.2.

\section{Resultados y discusión}

Se observó un mayor crecimiento y desarrollo de la variedad Dancy obteniendo la madurez de consumo en la lectura del día 222 después de antesis, lo que la clasificaría como de época de cosecha temprana, comparado con la variedad Arrayana y el genotipo LL053 que tuvieron un tiempo de madurez de consumo de 260 dda. Lo anterior permite considerar que la mandarina Dancy puede contribuir a ampliar la época de cosecha en el piedemonte Ilanero al poderse cosecharse un mes antes de la variedad regional Arrayana (Mateus y Orduz-Rodriguez en prensa)

\section{Peso de frutos}

Los frutos de los materiales evaluados presentaron una tendencia similar durante la fase de crecimiento (figura 1르). Sin embargo, se presentaron diferencias significativas entre materiales $(P<0,01)$ en todos los puntos de muestreo de la variable aumento de peso del fruto. El menor valor se obtuvo en el cultivar LL053, mientras que Arrayana presentó el mayor valor (figura 1A). Entre los días 188 y 208 dda se presentó una leve reducción en el aumento del peso de los frutos de los tres materiales evaluados; de acuerdo a Agustí et al. (2003) en este periodo se presentan todos los cambios asociados con la maduración de frutos. Este mismo comportamiento fue reportado para la mandarina Arrayana en estudios previos en la región (OrduzRodríguez et al., 2009) 


\section{Firmeza}

La importancia de la firmeza radica en que es considerada uno de los parámetros más importantes para determinar tanto la calidad como el efecto del manejo poscosecha y del empaque del producto (Kays, 1997). Para esta variable, se presentaron diferencias significativas entre materiales en todos los puntos de muestreo. Los mayores valores de firmeza se obtuvieron con el material LL053. Los materiales presentaron una tendencia parecida de la firmeza durante el crecimiento (figura 1B).
La disminución de la firmeza del fruto en el proceso de desarrollo se ocasiona por el proceso de hidrolisis de pectina de la pared celular, debido a la reducción del contenido de polisacáridos de la pared celular; proceso en el cual participan enzimas (Landanilla, 2008). La reducción de la firmeza durante el desarrollo y la maduración en mandarina también estás asociadas a cambios en los almidones, los cuales son especialmente abundantes en el albedo, aunque también están presentes en el flavedo. A medida que la fruta crece y madura, disminuyen por el metabolismo.

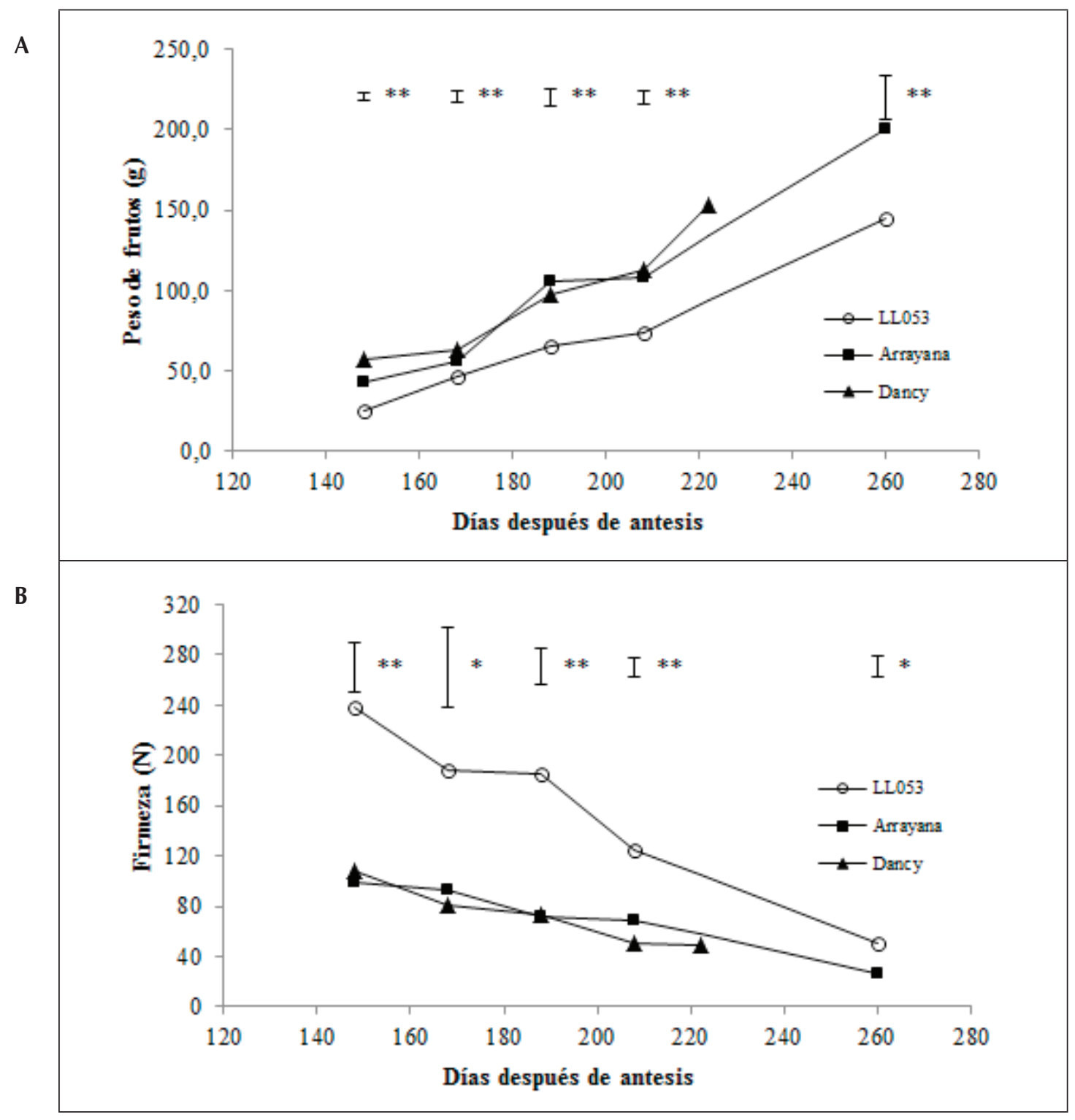

Figura 1. Comportamiento de: A. Peso de frutos (g) y B. Firmeza $(\mathrm{N})$ durante el crecimiento y desarrollo de los frutos de mandarina estudiados. La barra representa el valor estadístico de diferencia mínima significativa (LSD) para comparar los promedios, de acuerdo con la prueba de Tukey. Si las diferencias entre dos promedios en cada punto de muestreo son mayores al LSD, entonces habrá diferencia a un a de 0,05. ns: sin diferencias significativas.

* Diferencias significativas al 5\%, ${ }^{* *}$ Diferencias altamente significativas al 1\%. 
A

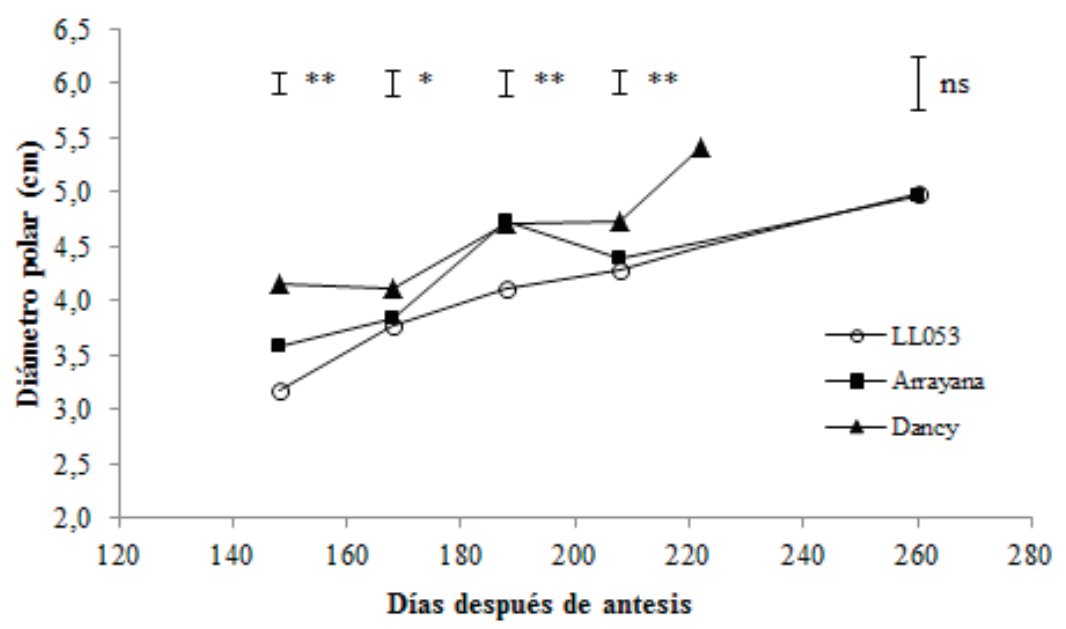

B

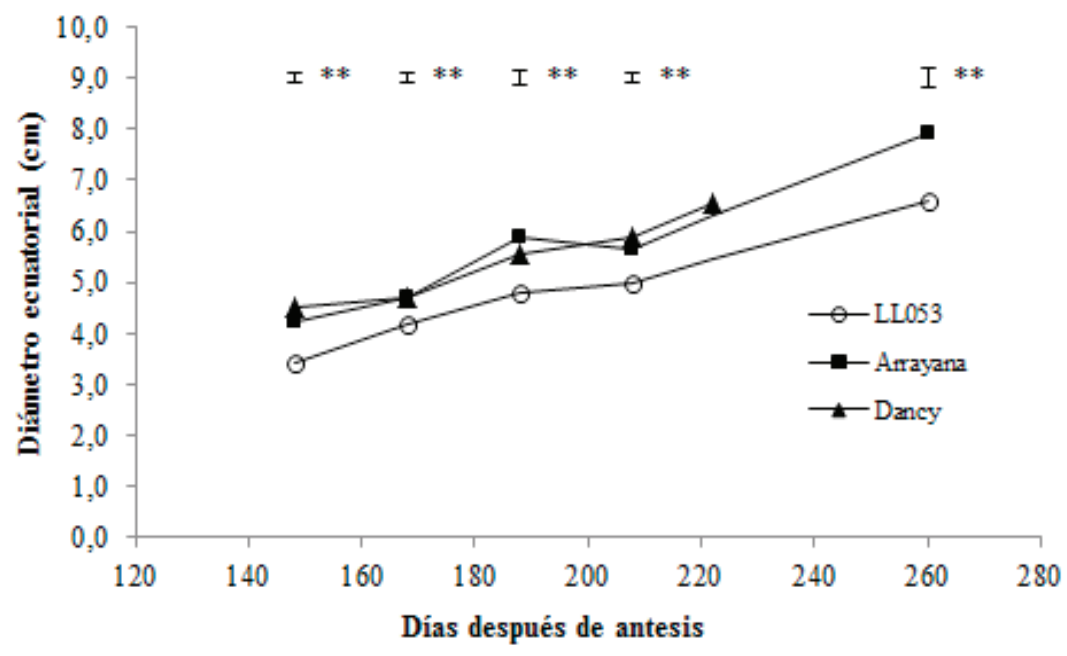

C

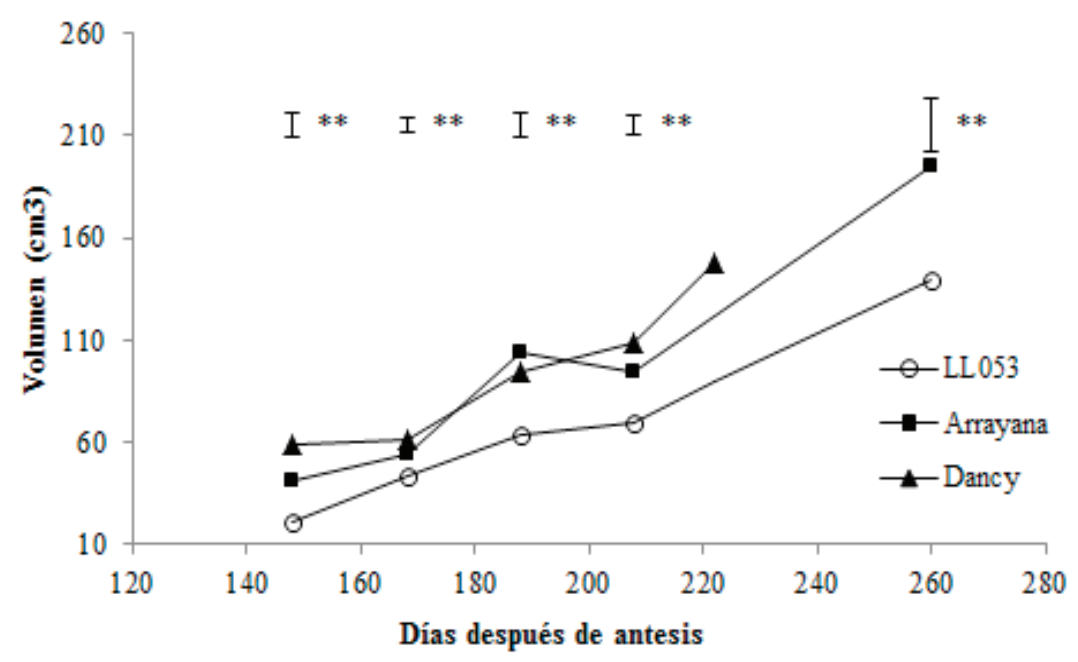

Figura 2. Comportamiento de: A. Diámetro polar $(\mathrm{cm})$ B. Diámetro ecuatorial $(\mathrm{cm})$ y C. Volumen $(\mathrm{cm} 3)$ de los frutos de mandarina estudiados durante su crecimiento y desarrollo. La barra representa el valor estadístico de diferencia mínima significativa (LSD) para comparar los promedios, de acuerdo con la prueba de Tukey. Si las diferencias entre dos promedios en cada punto de muestreo son mayores al LSD, entonces habrá diferencia a un a de 0,05. ns: sin diferencias significativas.

** Diferencias significativas al $5 \%,{ }^{* *}$ Diferencias altamente significativas al $1 \%$. 


\section{Diámetro polar, diámetro ecuatorial y volumen}

En los diámetros de los frutos se presentaron diferencias significativas entre materiales en todos los muestreos con excepción del último muestreo para diámetro polar (figura $2 \mathrm{~A}$ y $2 \mathrm{~B}$ ). Los tres materiales evaluados mostraron una tendencia similar durante el crecimiento. Respecto a volumen, en todos los puntos de muestreo se presentaron diferencias significativas entre materiales $(\mathrm{P}<0,01)$. A los 260 dda el mayor valor lo presentó en el material Arrayana, seguido por Dancy, mientras que el menor volumen de fruta lo obtuvo la mandarina LL053 (Figura 2C). Cabe resaltar que a los $222 \mathrm{dda}$, Dancy presentó los mayores valores de diámetro polar, diámetro ecuatorial y volumen, ya que este material presenta madurez de consumo por esa época. De acuerdo a Passaro et al. (2012), los frutos cítricos tienen alta relación superficie/volumen.

En general, el parámetro tamaño del fruto está asociado a las variables peso, diámetro ecuatorial y volumen (Bain, 1985). Según ellos, la mandarina Arrayana presentó los mayores valores en comparación con la mandarina Dancy y la mandarina LL053. Lo anterior podría explicar por qué la mandarina Arrayana es la variedad de mayor aceptación por el consumidor de Bogotá y la región central del país. Además del tamaño del fruto hay otros parámetros relacionados con la calidad, como el fácil pelado y el sabor, además de su buena adaptación a las condiciones climáticas de la región. Respecto a las mandarinas Dancy y LL053, aunque presentaron menores tamaños en este estudio, también pueden ser considerados frutos grandes con relación a las mandarinas Clementinas que pesan alrededor de $80 \mathrm{~g}$ y que son las de mayor exportación en el mercado mundial. Para la variedad Dancy, se reporta que la productividad media en terrazas altas del piedemonte del Meta está alrededor de las 30 t/ha (Orduz-Rodríguez y Mateus, 2014), 5 a 10 toneladas superior a la mandarina Arrayana, de acuerdo a la producción reportada por Orduz-Rodríguez, et al. (2012). Lo anterior sugiere el potencial que puede tener esta variedad para la región y para otras productoras en el trópico bajo del país.

\section{SST}

Hubo diferencias significativas a los 148, 168 y 208 dda. Durante el estudio los menores valores de SST se presentaron en Arrayana, por su parte la mandarina LL053 presentó el mayor valor de SST con $10.4^{\circ}$ Brix (Figura 3A).

Davies y Albrigo (1999) reportan que se presenta un leve incremento de los niveles de SST en la etapa final del desarrollo del fruto. En etapas tempranas los SST aumentan con el incremento del tamaño del fruto Agustí et al. (2003). Hardy y Sanderson (2010) mencionan que el contenido de sólidos solubles aumenta debido principalmente a la acumulación de sacarosa, en la fase de maduración. El mismo comportamiento fue reportado por Agustí et al. (2003), quienes señalan que en variedades con madurez temprana, el contenido en azúcares aumenta rápidamente y los frutos continúan su maduración cuando la temperatura desciende (en regiones subtropicales); pero en variedades tardías la maduración se da cuando la temperatura tiende a elevarse, y la sacarosa aumenta su contenido relativamente poco en el fruto (Agustí et al., 2003).

\section{ATT}

La acidez total titulable (ATT) en mandarina disminuyó durante su desarrollo de los tres materiales evaluados (Figura 3B). Los valores de acidez presentaron diferencias significativas en los diferentes tratamientos de todos los muestreos. Los valores encontrados están dentro del rango de acidez aceptable y además coinciden con los reportados por Cantuarias-Avilés et al. (2010) y Sánchez et al. (2008). De la misma forma, Cercós et al. (2006) reportan similar comportamiento en mandarinas Clementina, y atribuyen estos cambios a una disminución en la concentración de citrato (hasta 55\%). López y Cardona (2007) reportan que para naranja, el valor óptimo de ATT para la industria procesadora está entre 0,6 y $0,9 \%$. De la misma forma en España, el patrón Cleopatra ha producido frutos pequeños con altos valores de acidez (Fermino et al., 1997; Gomes, 1990; Castle et al., 1989).

De acuerdo a Davies y Albrigo (1994), los ácidos orgánicos contribuyen significativamente a la acidez total del jugo, siendo el ácido cítrico el ácido orgánico predominante (70-80\% del total). Los ácidos orgánicos son considerados una fuente importante de sabor ácido en la fruta y una fuente de energía en la célula vegetal (Landanilla, 2008). Losácidosgeneralmentedisminuyen durante la maduración, ya que ellos puedes ser utilizados como sustratos respiratorios o convertidos en azucares, aunque también se utilizan para la formación de compuestos aromáticos y del sabor (Cañizares et al., 2003; Landanilla, 2008). En la fase de maduración los ácidos libres disminuyen progresivamente como consecuencia, fundamentalmente, de un proceso de dilución (Agustí et al., 2003), lo cual sucede a medida que la fruta aumenta en tamaño y en contenido de jugo (Landanilla, 2008). Es importante señalar que la ATT es comúnmente utilizada como un componente para calcular el índice de madurez, más que como un parámetro independiente (Acevedo, 2008). 


\section{RM (SST/ATT)}

Los SST y la ATT son elementos importantes en la estimación del sabor y la calidad nutritiva de los cítricos (Li et al. 2012). La relación o índice de madurez (RM) se utiliza casi siempre para calcular la madurez mínima requerida en naranjas, mandarinas, toronjas y pomelos para el consumo en fresco (Passaro et al., 2012). En este estudio se observaron diferencias significativas $(\mathrm{P}<0,01)$, excepto en la madurez de consumo; se encontró que la RM incrementó durante el desarrollo (figura 3C). En la variedad Dancy se presentó una mayor velocidad en el aumento de la RM, los valores fueron mayores durante el desarrollo y maduración para este material, debido al mayor contenido de SST y a los menores valores de ATT. A los 208 dda presentó una RM de 10.38, mientras que el cultivar LL053 y la variedad Arrayana tuvieron 3.17 y 4.58 respectivamente. Aquello refleja la madurez de consumo temprana de la mandarina Dancy, al alcanzar valores superiores de la RM de consumo 6 semanas antes que las otras variedades.

Los principales cambios en la madurez están asociados con el aumento de los niveles de azúcar y la disminución de la acidez, por lo cual determinar el momento de cosecha adecuado para el consumo es fundamental (Hardy y Sanderson, 2010). Un equilibrio apropiado entre azúcares y ácidos de la fruta cítrica fresca hace que su sabor sea atractivo; la relación de madurez puede contribuir a determinar el punto óptimo para consumo, el vencimiento legal de la fruta, así como su palatabilidad (Landanilla, 2008).

\section{Índice de color}

Se observaron diferencias significativas $(P<0,01)$ entre la variedad Dancy y los otros dos materiales evaluados, excepto a los 208 dda. Los menores valores de IC durante el desarrollo y la maduración se presentaron en Arrayana y LL053; a los 208 dda se registró -13.73 y -8.84 de IC respectivamente. Por su parte, la variedad Dancy presentó -6.68. En madurez de consumo la variedad Dancy registró valores negativos, lo que equivale a un color entre verde y amarillo con una tendencia favorable al color naranja a los 220 dda (figura 4).

La luminosidad influye en la apariencia y en la percepción de calidad del fruto (Ariza et al., 2010), al igual que una relación positiva del IC. En la maduración hay una rápida síntesis de carotenoides acompañada de una pérdida simultánea de la clorofila (Landanilla, 2008). Esta se degrada a temperaturas menores de $15^{\circ} \mathrm{C}$ (Spiegel-Roy y Golschmidt, 1996) y los cloroplastos se convierten en cromoplastos que contienen pigmentos anaranjados y amarillos (carotenoides, licopenos, entre otros) (Spiegel-Roy y Golschmidt, 1996; Kato et al., 2004; Landanilla, 2008). En las condiciones del trópico, la clorofila no se degrada tanto como en condiciones subtropicales, debido principalmente a que no se presentan bajas temperaturas (Sdiri et al., 2011), y el color de la cáscara de algunos cítricos permanece verde incluso cuando el fruto está maduro internamente (Landanilla, 2008).

Según Huff (1984), la acumulación de azúcares en este tejido durante la maduración es el principal factor regulador de la coloración del fruto y tiene incidencia en la conversión de los plastidios, lo que indica que la disponibilidad por fotoasimilados del fruto puede ser un factor de maduración. Durante el desarrollo y la maduración, los cítricos cambian de verde a amarillo o naranja a rojo-naranja según el carácter genético y del crecimiento favorable de la variedad en las condiciones climáticas (Spiegel-Roy y Golschmidt, 1996; Landanilla, 2008).

\section{Características fisicoquímicas en madurez de consumo}

Se presentaron diferencias estadísticas $(P<0,05)$ entre materiales en todos los parámetros físicos y químicos en madurez de consumo, a excepción del diámetro polar y RM (Tabla 1). Los valores en Arrayana coindicen con lo reportado por Orduz-Rodríguez et al. (2009). Los valores de peso obtenidos en el presente ensayo fueron mayores a los reportados por OrduzRodríguez et al. (2011), en cuyo informe, la variedad Dancy y Arrayana presentaron un peso de 150 g y 159 $\mathrm{g}$, respectivamente.

En condiciones de subtrópico en La Florida, la mandarina Dancy es considerada un material con frutos pequeños, debido a que su peso varía entre 60 y 90 g (Landanilla, 2008). Por otro lado, los frutos de mandarina Arrayana producidos en el trópico, Departamento del Meta en Colombia, se clasifican como frutos grandes por presentar un peso promedio de 145 gramos, mayor a la variedad clementina (Orduz-Rodríguez et al. 2009).

De igual manera los frutos evaluados en el presente trabajo se pueden clasificar en frutos grandes, debido a que el peso promedio en madurez de consumo de la variedad Dancy y el cultivar LL053 representan el $76.5 \%$ y $72.5 \%$, respectivamente, del peso promedio de la variedad Arrayana (200 g). Probablemente, el mayor peso de los frutos producidos en el Departamento del Meta se deba a las condiciones climáticas que 
A

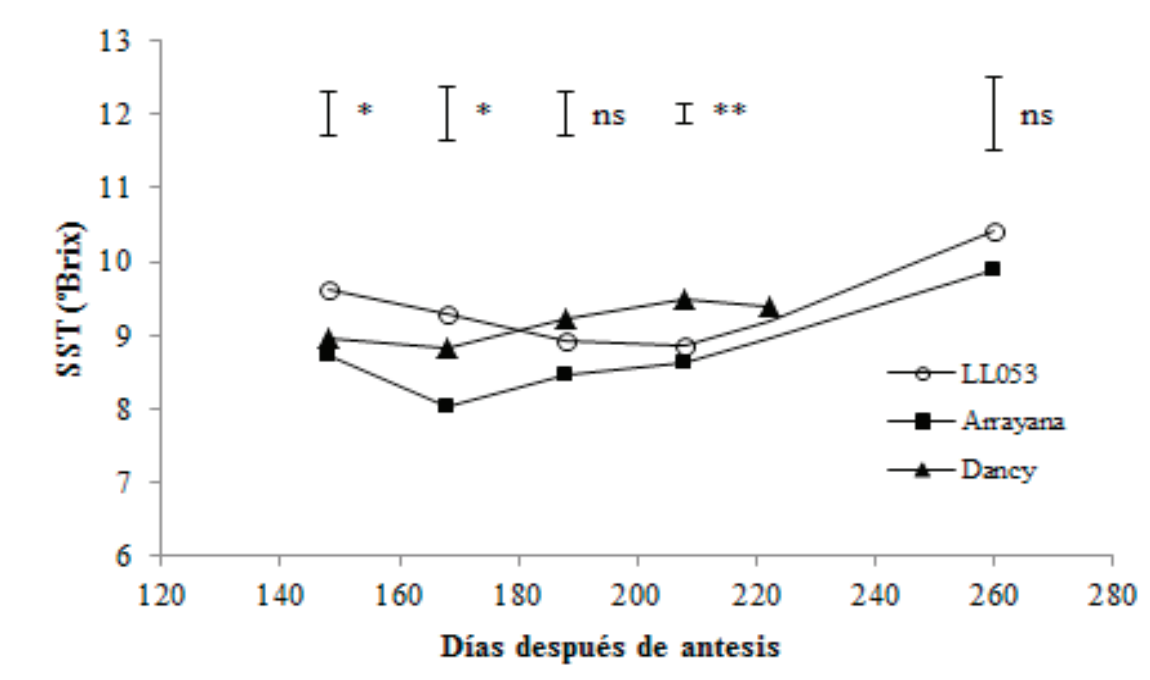

B

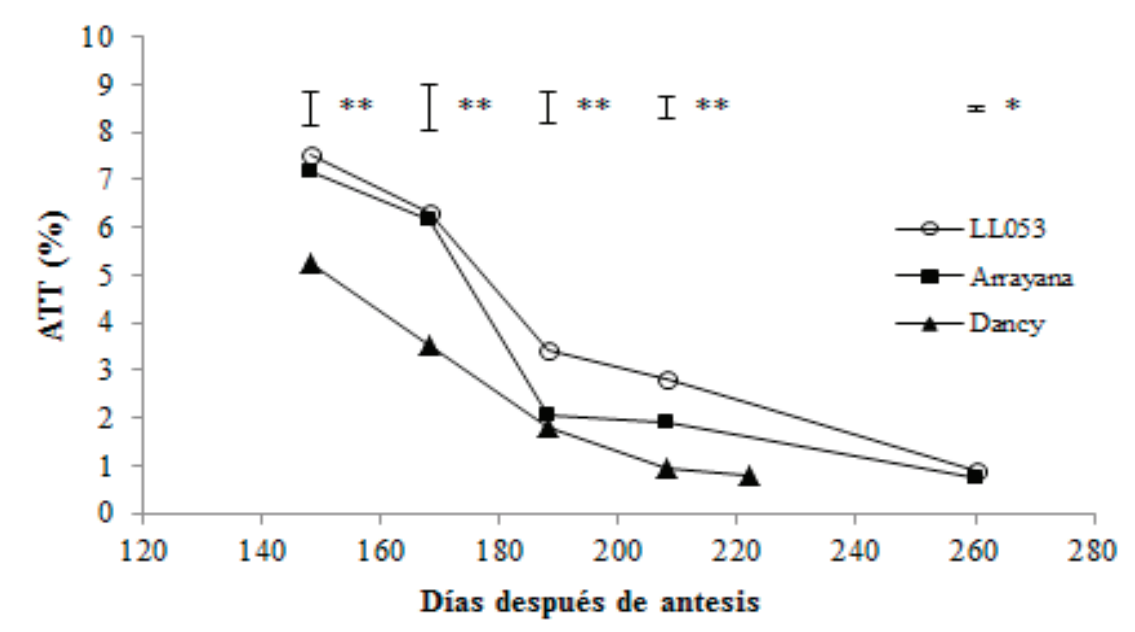

C

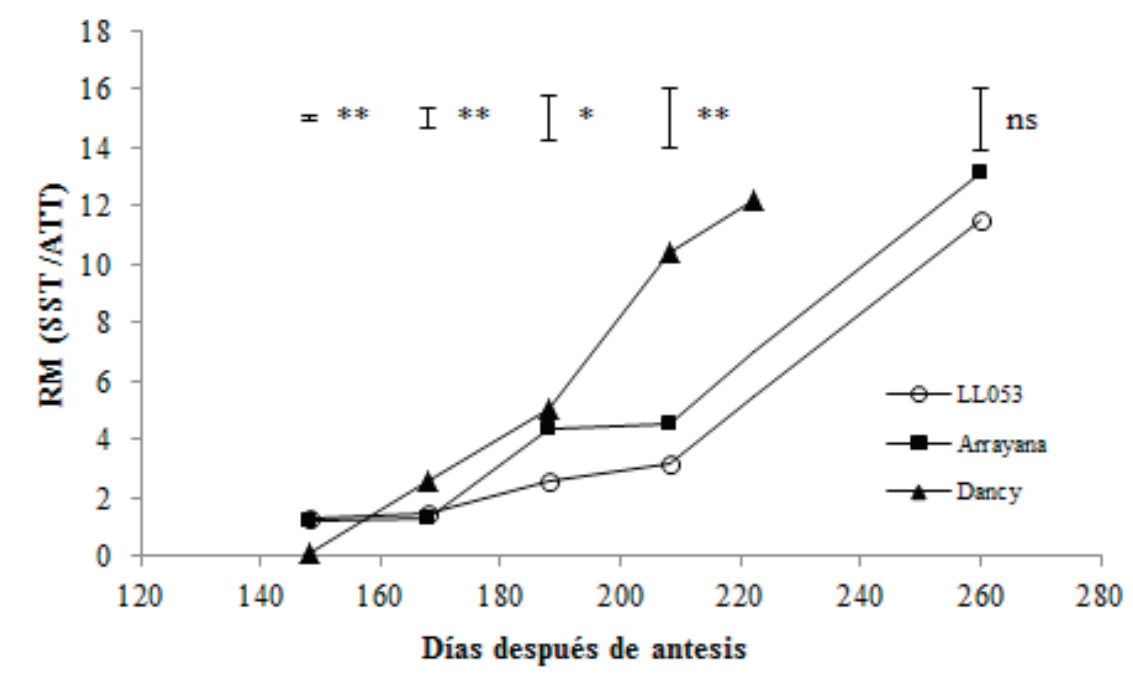

Figura 3. Comportamiento de: A. SST ( ${ }^{\circ}$ Brix), B. ATT (\%) y C. RM (SST/ATT) durante el crecimiento y desarrollo de los frutos de mandarina estudiados. La barra representa el valor estadístico de diferencia mínima significativa (LSD) para comparar los promedios, de acuerdo con la prueba de Tukey. Si las diferencias entre dos promedios en cada punto de muestreo son mayores al LSD, entonces habrá diferencia a un $\alpha$ de 0,05. ns: sin diferencias significativas.

*Diferencias significativas al $5 \%,{ }^{* *}$ Diferencias altamente significativas al $1 \%$. 


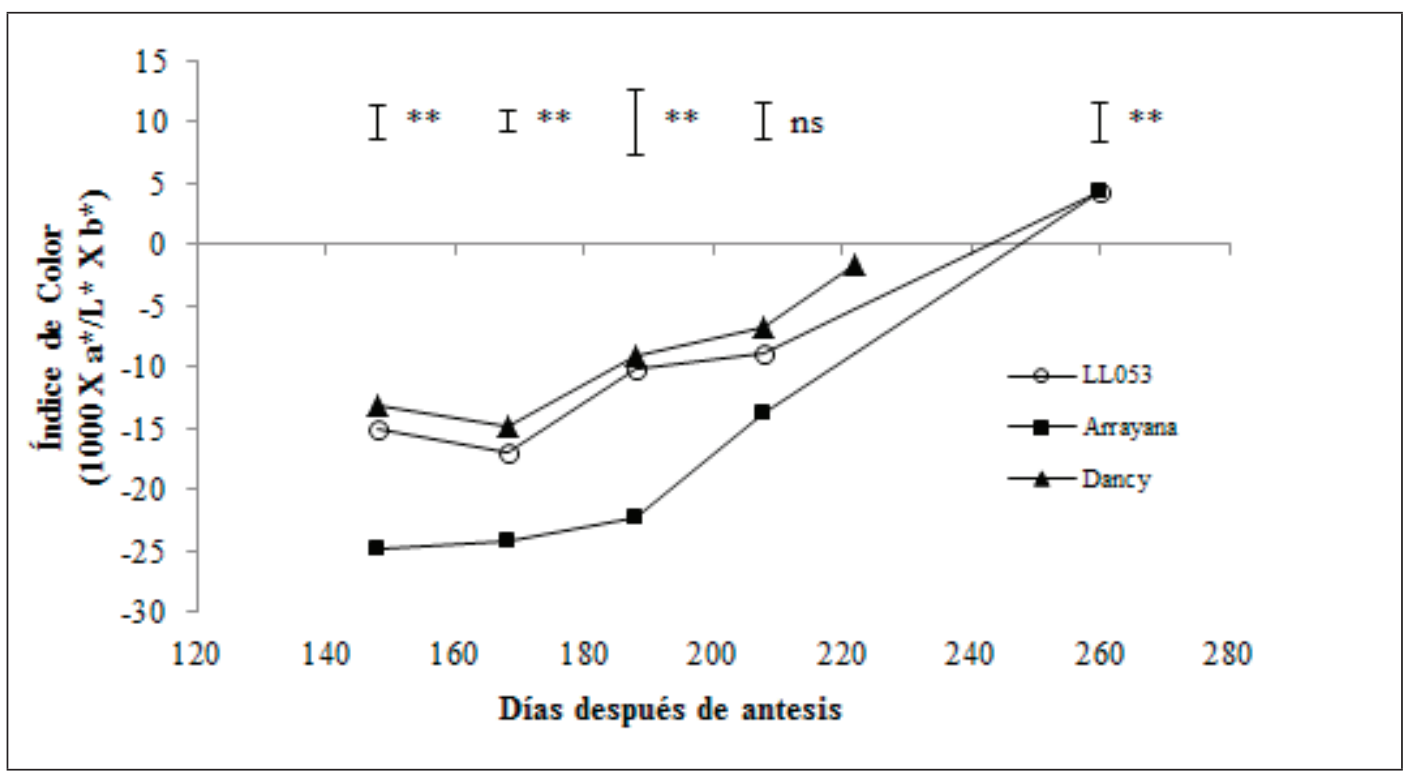

Figura 4. Comportamiento del Índice de color $\left(1000 \mathrm{X} \mathrm{a}^{*} / \mathrm{L}^{*} \mathrm{X} \mathrm{b}^{*}\right)$ en el crecimiento y desarrollo de los frutos de mandarina estudiados. La barra representa el valor estadístico de diferencia mínima significativa (LSD) para comparar los promedios, de acuerdo con la prueba de Tukey. Si las diferencias entre dos promedios en cada punto de muestreo son mayores al LSD, entonces habrá diferencia a un $\alpha$ de 0,05. ns: sin diferencias significativas, *Diferencias significativas al 5\%,**Diferencias altamente significativas al $1 \%$.
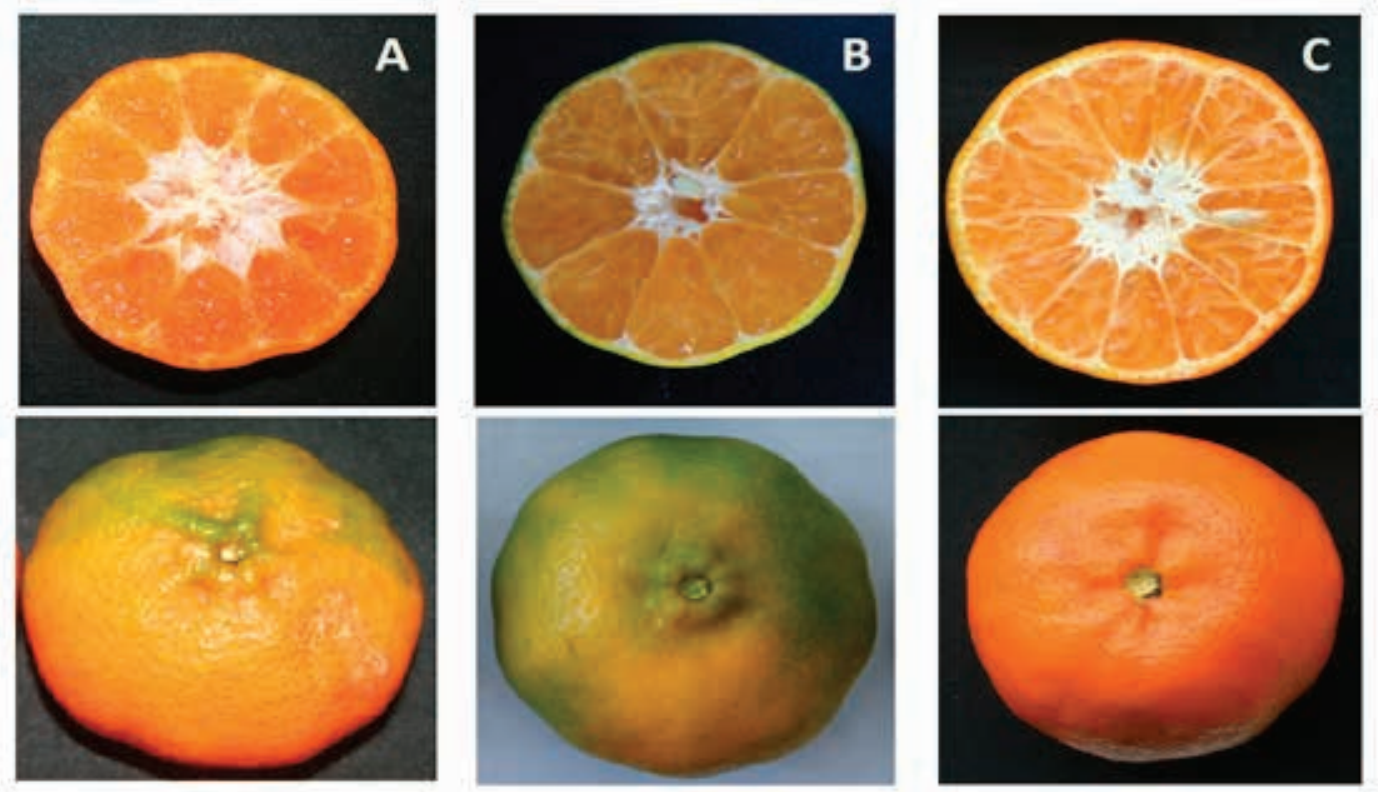

Figura 5. Frutos en madurez de consumo de mandarina A: Arrayana 260 dda, B: Dancy 222 dda y C: LL053 260 dda, cosechados en el Centro de Investigación La Libertad de Corpoica en Colombia.

posee la región para la acumulación de materia seca, en especial por la precipitación y la acumulación de unidades de calor anual, teniendo en cuenta que los cítricos acumulan cerca de 5000 unidades de calor anual (Orduz-Rodríguez, 2007).

Se observaron diferencias significativas de volumen entre la variedad Arrayana y los otros dos materiales evaluados. Pese a que la variedad Dancy no presentó diferencias respecto al cultivar LL053, presentó mayor volumen (Tabla 1). El volumen de mandarina Arrayana fue mayor al reportado por Orduz-Rodríguez et al. (2009), donde alcanzó un promedio de $161.8 \mathrm{~cm}^{3}$.

La relación peso/volumen es un indicador de la calidad interna del fruto; qué tan jugoso o tan seco está el fruto 
(Pássaro et al., 2012). Se encontró que la relación peso/volumen de las mandarinas Arrayana, Dancy y LL053 fue de 1.032, 1.033 y 1.040 respectivamente, sugiriendo mayor jugosidad en esta última.

Se observaron diferencias estadísticas entre los valores de diámetro ecuatorial entre Arrayana y los demás materiales (Dancy y LL053). El mayor diámetro ecuatorial se presentó en frutos de Arrayana (Tabla 1). Orduz-Rodríguez et al. (2009) reportan un diámetro ecuatorial promedio de Arrayana de $7.5 \mathrm{~cm}$, valor que coincide con este trabajo. Sin embargo, los valores promedio del diámetro polar no presentaron diferencias estadísticas entre los materiales de mandarina evaluados. El diámetro polar de Arrayana coincide con los resultados encontrados por OrduzRodríguez et al., (2009), donde el valor promedio fue de 5,4 cm. Datos del mismo autor y para la misma variedad indican una relación altura/diámetro de 0.72, en su madurez. La relación altura/diámetro en el presente estudio para Arrayana, Dancy y LL053 fue de $0.6,0.8$ y 0.7 , respectivamente.

Hubo diferencias estadísticas entre Arrayana y los otros dos materiales en cuanto a la firmeza de los frutos. Los mayores valores de firmeza se presentaron en la variedad Dancy y el cultivar LL053, lo que significa que el menor valor se presentó en mandarina Arrayana (Tabla 1). Las frutas que presentan menor firmeza son más susceptibles a deteriorarse durante el manejo poscosecha (Arias y Toledo, 2000). La firmeza como indicador de maduración de frutos refleja los niveles de calidad para el consumo y está relacionada con las condiciones de transporte y manejo poscosecha del producto (Barbosa-Canovas et al. 2003).

La presencia de semillas en los frutos es una característica no deseada por los consumidores, por lo que se han desarrollado muchos trabajos de mejoramiento genético en cítricos tratando de disminuir su número o incluso, eliminar su presencia (Pássaro et al., 2012). Se observaron diferencias estadísticas entre los valores de número de semillas de la mandarina Arrayana y la mandarina Dancy (Tabla1). La mandarina Arrayana presentó los menores valores en número de semillas (10.1 \pm 1.6$)$, seguido por LL053 (12.7 \pm 1$)$, mientras que la variedad Dancy presentó los mayores valores en número de semillas $(16.0 \pm 0.8)$.

En contenido de jugo se observó diferencias estadísticas entre los valores promedios de mandarina Arrayana y los materiales Dancy y LL053 (Tabla 1). Estas últimas presentaron en promedio un $10 \%$ más de contenido que la mandarina Arrayana. De acuerdo a Orduz-Rodríguez et al. (2006), los contenidos de jugo se consideran altos para mandarina cuando son superiores a $27.4 \%$ (valor registrado para mandarina Ponkan). De igual forma, para naranjas y tangelos el porcentaje mínimo aceptado en frutos destinados para la elaboración de jugo debe ser superior al 40\% (Orduz-

Tabla 1. Características fisicoquímicas evaluadas en madurez de consumo en los frutos de mandarinas investigadas. Promedios seguidos de letras diferentes en la misma fila presentan diferencias estadísticas según la prueba de Tukey $(p \leq 0,05)$.

\begin{tabular}{|c|c|c|c|}
\hline \multirow{2}{*}{ Parámetros } & \multicolumn{3}{|c|}{ Cultivar-Variedad } \\
\hline & Arrayana & Dancy & Cultivar LL053 \\
\hline $\begin{array}{l}\text { Días después de antesis sobre los que se } \\
\text { evaluaron los parámetros }\end{array}$ & 260 & 222 & 260 \\
\hline \multicolumn{4}{|l|}{ Físicos } \\
\hline Peso (g) & $200.5 \pm 10.1 \mathrm{a}$ & $152.6 \pm 5.5 b$ & $144.5 \pm 6.1 b$ \\
\hline Volumen $\left(\mathrm{cm}^{3}\right)$ & $194.3 \pm 10 \mathrm{a}$ & $147.7 \pm 5.7 b$ & $139.0 \pm 6.2 b$ \\
\hline Diámetro ecuatorial $(\mathrm{cm})$ & $7.9 \pm 0.2 \mathrm{a}$ & $6.5 \pm 0.3 b$ & $6.6 \pm 0.2 b$ \\
\hline Diámetro polar $(\mathrm{cm})$ & $4.9 \pm 0.37 \mathrm{a}$ & $5.4 \pm 0.3 \mathrm{a}$ & $4.9 \pm 0.2 \mathrm{a}$ \\
\hline Número de semillas & $10.1 \pm 1.6 \mathrm{~b}$ & $16.0 \pm 0.8 \mathrm{a}$ & $12.7 \pm 1 \mathrm{ab}$ \\
\hline Firmeza $(\mathbf{N})$ & $26.46 \pm 0.98 b$ & $54.88 \pm 4.9 \mathrm{a}$ & $49.98 \pm 5.8 \mathrm{a}$ \\
\hline Índice de color (1000 X a*/L* X b*) & $4.292 \pm 0.31 \mathrm{a}$ & $-1.538 \pm 0.1 b$ & $4.293 \pm 0.35 \mathrm{a}$ \\
\hline Contenido de jugo (\%) & $30.8 \pm 1.1 \mathrm{~b}$ & $40.8 \pm 1.6 \mathrm{a}$ & $40.7 \pm 3 \mathrm{a}$ \\
\hline \multicolumn{4}{|l|}{ Químicos } \\
\hline SST $\left({ }^{\circ}\right.$ Brix $)$ & $9.8 \pm 0.06 \mathrm{ab}$ & $9.4 \pm 0.2 b$ & $10.4 \pm 0.3 \mathrm{a}$ \\
\hline $\mathrm{ATT}(\%)$ & $0.75 \pm 0.01 \mathrm{~b}$ & $0.77 \pm 0.03 b$ & $0.91 \pm 0.03 a$ \\
\hline pH & $4.2 \pm 0.04 \mathrm{a}$ & $3.7 \pm 0.07 \mathrm{~b}$ & $3.5 \pm 0.04 b$ \\
\hline RM (SST/AAT) & $13.1 \pm 0.2 \mathrm{a}$ & $12.2 \pm 0.5 \mathrm{a}$ & $11.5 \pm 0.7 \mathrm{a}$ \\
\hline
\end{tabular}


Rodríguez et al., 2011). Para el caso de mandarina en el presente ensayo los valores correspondientes a contenido de jugo fueron superiores al $40 \%$ para la variedad Dancy y el cultivar LL053, los cuales se pueden considerar valores altos.

En SST se presentaron diferencias estadísticas entre la mandarina Dancy y la LL053. El mayor contenido de SST se presentó en mandarina LL053 cuyo valor estuvo $0.6{ }^{\circ}$ Brix por encima de la mandarina Arrayana y $1.0^{\circ}$ Brix más que la variedad Dancy. Al respecto, Hagenmainer y Baker (2004) afirman que el sabor de los frutos está relacionado con los SST. El valor de $8.13{ }^{\circ}$ Brix de la mandarina Arrayana se considera moderado respecto al valor de 15.73 -Brix alcanzado por la mandarina Fortune, citado por Orduz-Rodríguez et al. (2009). Del 75 al 85\% de los sólidos solubles totales en zumos de naranja, mandarina, pomelos y limones son azúcares entre los que se destacan, sacarosa, glucosa y fructosa, con proporción 2:1:1 (Agustí et al., 2003).

Se observaron diferencias estadísticas entre los valores promedios de ATT, entre la mandarina LL053 $(0.91 \pm 0.03)$ y los otros dos materiales evaluados. $(0.75 \pm 0.01$ para Arrayana y $0.77 \pm 0.03$ para Dancy) (Tabla 1). Lo anterior coincide con lo reportado por Orduz-Rodríguez et al. (2011), quienes reportan un valor entre $0.82 \%$ y $1.0 \%$ de ATT para la variedad Dancy.

En la RM no se observaron diferencias estadísticas entre los valores de mandarina Arrayana. Los valores aceptables de relación de madurez para cosecha son de 9 en adelante. (Orduz-Rodríguez et al., 2009; Orduz-Rodríguez et al., 2011), aunque, de acuerdo a Orduz-Rodríguez y Avella (2008), los mejores valores para el consumo de la fruta están entre un índice de madurez de 10 hasta 13, es decir que los tres materiales evaluados tienen un índice de madurez adecuado al momento de cosecha Arrayana 13.1 \pm 0.2 ; LL053 11.5 \pm 0.7 ; Dancy 12.2 \pm 0.5 ; con un RM de 10. Arrayana podría cosecharse desde aproximadamente los 252 dda, la LL053 desde los 244 dda y Dancy desde los 208 dda.

Para el IC se observaron diferencias estadísticas entre los materiales. Los mayores índices de color en madurez de consumo se presentaron en la variedad Arrayana y el cultivar LL053, mientras que la variedad Dancy presentó los frutos más verdes. Es importante observar en la figura 4 que Dancy muestra una tendencia a obtener un color naranja más rápidamente. Al día 222 dda Dancy alcanzó un IC de -2, mientras que Arrayana se encontró en -9 y LL053 en -6.
Dancy podría brindar una gran ventaja a los agricultores de la región al alcanzar su madurez de consumo casi un mes antes que Arrayana, con unas características de calidad de fruto adecuadas que permitiría descentralizar la cosecha que actualmente se da en los meses de diciembre y enero. Si los frutos se dejan más tiempo en el árbol, el color externo de su cáscara alcanzará un color más atractivo (OrduzRodríguez y Mateus, 2014); esas condiciones le permitiría competir en condiciones de igualdad con las mandarinas importadas.

\section{Conclusiones}

El material "Dancy" presentó una maduración temprana; el tiempo de madurez de consumo se alcanzó a los 222 dda, mientras que Arrayana y LL053 tuvieron un tiempo de formación de 260 dda.

En general, en madurez de consumo los frutos de la variedad Arrayana, presentaron los mayores valores de peso, diámetro ecuatorial y pH. El cultivar "LL053" y la variedad "Dancy" exhibieron altos valores en contenido de jugo (10\% más que Arrayana) y firmeza. El cultivar LL053 obtuvo el mayor porcentaje de ATT con $0.91 \pm 0.03$ y Arrayana el menor con $0.75 \pm$ $0.01 \%$. El mayor valor de SST se presentó en el cultivar LL053, mientras que la variedad Dancy presentó los menores valores.

De acuerdo a la Relación de Madurez (RM) en el tiempo la mandarina Dancy se clasifica como una mandarina de época de cosecha temprana en condiciones tropicales, lo que podría permitir ampliar la época de cosecha del cultivo de mandarina en el Piedemonte Ilanero y mejorar la rentabilidad de las fincas citrícolas de la región.

\section{Agradecimientos}

Los autores agradecen la colaboración de la Corporación para la Investigación Agropecuaria Corpoica, el Instituto de Ciencia y Tecnología de Alimentos de la Universidad Nacional de Colombia y la Facultad de Ciencias Agrarias. Así mismo, se quiere destacar el apoyo del Ingeniero Agrónomo Fredy Alexander Salamanca en la edición de este documento.

\section{Referencias}

Acevedo YP. 2008. Eventos fisiológicos asociados a la madurez y calidad de frutos cítricos en Cuba y su relación con los productos transformados de la industria. Instituto de Investigaciones en Fruticultura Tropical. FAO: $1-21$ 
Agustí M, Martínez-Fuentes A, Mesejo C, Juan M, Almela V. 2003. Cuajado y Desarrollo de los Frutos Cítricos. GENERALITAT VALENCIANA. Serie Divulgación Técnica No. 55:1-82.

Anderson C. 1996. Variedades cultivadas en el Río Uruguay. En: Manual para productores de mandarina de la región del Río Uruguay. INTA, Buenos Aires, Argentina, pp. 63-91.

Arana JA. 2013. Juntos construimos una región líder en agricultura ecoeficiente. Boletín informativo. Ciencia, Tecnología e innovación, El futuro de la Orinoquia Colombiana. $6 p$.

Arias CJ, Toledo J. 2000. Manual de manejo poscosecha de frutas tropicales (papa, piña, plátano, cítricos). Aspectos Generales. "Técnicas mejoradas de poscosecha, procesamiento y comercialización de frutas". FAO.

Ariza R, Tejacal I, Beltrán M, Cervantes R, Alonso A, Barrios A, Moreno F. Calidad de los frutos de naranja 'Valencia' en Morelos, México. Revista Iberoamericana de Tecnología Postcosecha, 2010; 11(2):148-153.

Bain J. Morphological, anatomical and physiological changes in the developing fruit of the Valencia orange, $\mathrm{Ci}$ trus sinensis L. Osbeck. Australian Journal of Botanic, 1985; 6: 1-24.

Barbosa-Cánovas B, Fernandez-Molina J, Alzamora S, Tapia M, López-Malo A, Chanes J. 2003. Handling and preservation of fruits and vegetables by combined methods for rural areas. Technical Manual FAO Agricultural Services Bulletin, no. 149. Roma. 99 p.

Cantuarias-Avilés T, Alves F, Sanches E, Rodrigues S, Espinoza-Núñez E. Tree performance and fruit yield and quality of "Okitsu" Satsuma mandarin grafted on 12 rootstocks. Scientia Horticulturae, 2010; 123: 318-322

Cañizares A, Puesme R, Laverde D. 2003. Determinación de la curva de crecimiento y desarrollo del fruto de la lima persa (Citrus latifolia Tanaka) en el estado de Monagas. XV Congreso Venezolano de Botánica. pp:54-55

Castle W, Tucker D, Krezdorn A, Youtsey C. 1989. Rootstocks for Florida citrus. Institute of Food and Agricultural Sciences-University of Florida, Gainesville. 47p.

Cercós M, Soler G, Iglesias DJ, Gadea J, Forment J, Talón M. Global analysis of gene expression during development and ripening of citrus fruit flesh. A proposed mechanism for citric acid utilization. Plant Mol Biol, 2006; 62: 513-527.

Davies FS, Albrigo LG. 1994. Citrus. CAB International, Wallingford, U.K. 254p.

Davies, F. S. y L.G. Albrigo. 1999. Citricos. CAB international. Wallingford, U.K. Editorial Acribia S.A. 283 p.

Díaz JF. 2000. Caracterización de frutos de mangostino (Garcinia mangostana L.) y uso de envolturas plásticas para su conservación. Universidad Nacional de Colombia. Facultad de Agronomía. Bogotá.

Fermino E, Sanches E, Donadio L. 1997. Porta-enxertos para a citricultura Paulista. Jaboticabal: Funep. (Boletim Citrícola, 1) 47p.
Gomes K. 1990. Avaliação de cavalos tolerantes à tristeza enxertados em laranja Valencia. In: seminário internacional de citros, 85p.

Hagenmainer RD, Baker RA. 2004. Quality of fresh citrus fruit. Quality of fresh and processed foods. Kluwer academic.

Hardy S, Sanderson G. 2010. Citrus maturity testing. Prime fact 980:1-6.

Iglesias D, Cercos M, Colmeneros-Flores J, Naranjo M, Ríos G, Carrera E, Ruiz-Rivero O, Liso I, Orillon RM, Tadeo F, Talon M. Physiology of citrus fruiting. Plant Physiology, 2007; 19(4): 333-362.

Huff A. Sugar regulation of plastid interconversions in epicarp of citrus fruit. Plant Physiol, 1984; 76: 307-312.

Kato M, Ikoma Y, Matsumoto H, Sugiura M, Hyodo H, Yano $M$. Accumulation of carotenoids and expression of carotenoids biosynthetic genes during maturation in citrus fruit. Plan physiology, 2004; 134: 824-837.

Kays S. 1997. Postharvest physiology of perishable plant products. Exon Press. Athens, GA, 532p.

Landanilla MS. 2008. Fruit morphology, anatomy and phisiology. Capitulo 2-7.Citrus fruit. Biology, Tecnhonolgy, and Evaluation. First edition. Academic Press. India.

Li Q, Wu F, Li T, Su X, Jiang G, Qu H, Jiang Y, Duan X. Methylcyclopropene extends the shelf-life of 'Shatangju' mandarin (Citrus reticulate Blanco) fruit with attached leaves. Postharvest Biol Tec, 2012; 67: 92-95.

López J, Cardona J. 2007. Evaluación de portainjertos de cítricos en la zona central cafetera de Colombia. Cenicafé. Caldas, Colombia. 55p.

Morales J, López J. 2007. Aspectos básicos para el cultivo de mandarina (Citrus reticulata). Manual técnico. Bogotá: Corpoica; Produmedios.

Orduz-Rodríguez JO. Enfoque de la investigación de cítricos en los Ilanos orientales de Colombia. Opinión. Revista Achagua, 2003; 7(9): 3-6.

Orduz-Rodríguez JO, Arango-Wiesner L, Monroy H, Fischer G. Comportamiento de la mandarina Arrayana en seis patrones en suelos ácidos del piedemonte Llanero de Colombia. Agronomía Colombiana, 2006; 24(2): 266-273.

Orduz-Rodríguez JO. 2007. Estudios ecofisiológicos y caracterización morfológica y molecular de la mandarina 'Arrayana' (Citrus reticulata Blanco) en el piedemonte Ilanero de Colombia. Tesis doctoral. Facultad de Agronomía, Universidad Nacional de Colombia, Bogotá.

Orduz-Rodríguez JO. 2008. Perspectivas y posibilidades del cultivo de cítricos en el departamento de Casanare. Características de la citricultura del departamento del Casanare y recomendaciones para su mejoramiento productivo. Boletín de Investigación. CORPOICA C.I. La Libertad. pp 8-13.

Orduz-Rodríguez JO, Avella F. Comportamiento de 26 cultivares de naranja en condiciones del piedemonte del Meta, Colombia. Rev Col Cienc Hort, 2008; 2:157-172.

Orduz-Rodríguez JO, Monroy H, Fisher G, Herrera A. Crecimiento y desarrollo del fruto de mandarina (Citrus 
reticulata) 'Arrayana' en condiciones del piedemonte del Meta, Colombia. Rev Col Cienc Hort, 2009; (2): 149-160.

Orduz-Rodríguez JO, Castiblanco S, Calderón C, Velásquez $\mathrm{H}$. Potencial de rendimiento y calidad de 13 variedades e híbridos comerciales de cítricos en condiciones del piedemonte Ilanero de Colombia. Rev Col Cienc Hort, 2011; 5 (2):171-185.

Orduz-Rodríguez JO, Mateus D. 2012. Generalidades de los cítricos y recomendaciones agronómicas para su cultivo en Colombia. Capítulo 2. Cítricos: Cultivo, poscosecha e industrialización. Serie Lasallista Investigación y Ciencia, p 49-88.

Orduz-Rodríguez JO, Monroy J, Barrera S, Nuñez V, Ligarreto G. Caracterización morfo-agronónomica y molecular de mandarina 'Arrayana' en el piedemonte del Meta (Colombia). Revista Corpoica - Ciencia y tecnología agropecuaria, 2012; 13(1):5-12.

Orduz-Rodríguez JO, Mateus D. 2014. Mandarina Dancy: una nueva alternativa para la citricultura del piedemonte Ilanero de Colombia. Revista Corpoica. En prensa.

Pássaro C, Navarro P, Salvador A. 2012. Poscosecha. Capítulo 9. Cítricos: Cultivo, poscosecha e industrialización. Serie Lasallista Investigación y Ciencia, 370 p.

Sanches E, Espinoza F, Alves F, Marcos E. Vigor, produtividade e qualidade de frutos de quatro tangerineiras e híbridos sobre quatro porta-enxertos. Rev. Bras. Frutic., Jaboticabal - SP, 2008; 30(3): 741-747.

Sdiri S, Navarro P, Monterde A, Benabda J, Salvador A. New degreening treatments to improve the quality of citrus fruit combining different periods with and without ethylene exposure. Postharvest Biol Tec, 2011; 63: 24-32.

Sdiri S, Navarro P, Monterde A, Benebda J, Salvador A. Effect of postharvest degreening followed by a cold-quarantine treatment on vitamin $C$, phenolic compounds and antioxidant activity of early-season citrus fruit. Postharvest Biol Tec, 2012; 65: 13-21.

Spiegel-Roy P, Golschmidt EE. 1996. Biology of Citrus. Cambridge University Press, Cambridge, UK. 